\title{
Exogenous Application of Thiourea Ameliorates Salt Stress Effects by Alleviation of Oxidative Damage in Hybrid Maize
}

\author{
Tayyaba Sanaullah ${ }^{1}$, Abdul Wahid ${ }^{1}$, Bushra Sadia ${ }^{2}$, Asma Hanif ${ }^{1}$, Nazimah Maqbool ${ }^{1}$, Taskeen Arshad $^{1}$ and \\ Zohra Kabir ${ }^{1}$ \\ 1. Department of Botany, University of Agriculture, Faisalabad 38040, Pakistan \\ 2. U.S.-Pakistan Center for Advanced Studies in Agriculture and Food Security (USPCAS-AFS), University of Agriculture, Faisalabad \\ 38040, Pakistan
}

\begin{abstract}
Salinity is the most damaging factor towards crop production. The aim of this study was to investigate the possible role of thiourea in salinity tolerance. These experiments were carried out on two maize hybrids (DK6789 and 33M15) using the optimized thiourea level $(400 \mu \mathrm{M})$ in autumn and spring seasons, and the growth and physiological attributes were observed. Salt stress caused a substantial suppression of growth, and damaging effects were observed, as reduction in shoot and root length, their fresh and dry weights, decreased root number and shoot/root ratio, increased accumulation of $\mathrm{H}_{2} \mathrm{O}_{2}$, malondialdehyde (MDA) in root and shoot tissues, and enhancement in activities of some key antioxidant enzymes (catalase, peroxidase and superoxide dismutase) with altered ascorbate peroxidase level in leaves of both maize hybrids. Medium supplemented thiourea was found to be very effective in improving growth may be due to decreased $\mathrm{H}_{2} \mathrm{O}_{2}$, MDA and further enhanced the activities of antioxidants with higher accumulation of ascorbate peroxidase under control and saline conditions. Salinity tolerance induced by thiourea was superior in the spring than in autumn grown maize. From the changes in the growth and physiological attributes of maize, it can be concluded that thiourea application has a great potential to alleviate the deteriorating effects of salt stress on maize and is recommended for improving growth in marginally to moderately saline soils.
\end{abstract}

Key words: Salinity, thiourea, malondialdehyde, $\mathrm{H}_{2} \mathrm{O}_{2}$, maize.

\section{Introduction}

Increased levels of soluble salts in soil cause an unescapable threat for growth of plants by disturbing normal growth and metabolism [1]. As the salinity level increases, the growth and productivity decrease [2]. Saline conditions impose negative effects on plant height, biomass and yield [3]. The capability to reduce the damaging effects of reactive oxygen species (ROS) on key macromolecules in plant tissues is of great importance in stress tolerance [4]. To limit oxidative damage in stress condition, plants have developed a series of detoxification systems that breakdown toxic ROS [5]. A positive correlation between antioxidant

Corresponding author: Tayyaba Sanaullah, Ph.D., research field: botany. and salinity stress tolerance has been investigated in sunflower [6], turnip [7], wheat [8] and pea [9].

Exogenous application of organic and inorganic compounds proved to be very useful to improve crop salt tolerance [10]. Thus, application of bio-regulatory hormones has the potential to provide pragmatic and easy solutions to ensure sustainable agriculture in salt affected lands in future [11]. It has been shown that a number of natural and synthetic plant growth regulators, inorganic salts and stress signaling molecules can be used to improve stress tolerance in plants depending upon their specific properties, mechanisms of action and their roles to improve germination $[12,13]$.

Thiourea is an important growth regulator and is 
thought to be involved in improving stress tolerance in crops [13]. It is water soluble and can easily penetrate plant cells. Structurally, thiourea has three functional groups, i.e., "thiol", "amino" and "imino" groups [14]. Oxidative stress can be tolerated by thiol group, and amino and imino groups help to partially fulfill the $\mathrm{N}$ economy when plant is facing abiotic stress [15]. Seasons also affect the growth and yield of crops, because different seasons have different temperatures. Application of thiourea at late sowing dates increased the yield of Brassica [16]. Thiourea also improved the growth and yield of wheat under salinity and high temperature stress [17] and improved cadmium tolerance in maize when supplemented in the growth medium [14].

Salt stress reduces the growth by affecting various morphological and physiological attributes in maize [18]. Salt stress causes oxidative damage that can be determined in terms of the accumulation of malondialdehyde (MDA) and $\mathrm{H}_{2} \mathrm{O}_{2}$ [19]. Superoxide dismutase (SOD), catalase (CAT) and peroxidase (POD) are the important antioxidant enzymes, because they can efficiently prevent the accumulation of $\mathrm{O}^{2-}$ and $\mathrm{H}_{2} \mathrm{O}_{2}$ and minimize the deleterious effects of ROS [20]. Though, the plants can escape the destructive effects of MDA and $\mathrm{H}_{2} \mathrm{O}_{2}$ by developing a strong defense system of antioxidant enzymes, like CAT, POD and SOD [21]. They seem to be important for plant development, and the enhanced growth and yield have been achieved [22].

One of the important effects of salinity on plant cells and tissues is the enhanced production of ROS [23], which damages the biological membranes. In order to reduce the effect of salinity, it is important to reduce the production of ROS in plant tissues. Most of the studies in literature deal with the effectiveness of foliar spray of thiourea in mitigating the stress affect. It is perceived that the medium supplementation of thiourea is an excellent source to mitigate the ROS production due to its important structural properties. The objective of this study was to assess the possible role of thiourea in the activation of antioxidants, reducing production of ROS and improvement in growth of salinity stressed hybrid maize, a commercially important crop.

\section{Materials and Methods}

\subsection{Plant Materials}

Experiments were conducted in the autumn and spring seasons of 2013-2014 in the Department of Botany, University of Agriculture, Faisalabad, Pakistan. Seeds of DK6789 and 33M15 (single cross hybrids) were obtained from Maize and Millet Research Institute, Sahiwal. Seeds were sown in plastic pots containing river sand. An optimized level $(400 \mu \mathrm{M})$ of thiourea and $120 \mathrm{mM} \mathrm{NaCl}$ salinity were applied to 10-day-old plants mixed in half strength nutrient solution [24]. After 15 days of treatment, the data was taken for growth attributes, and plant were preserved in freezer at $-30{ }^{\circ} \mathrm{C}$ for different analysis. Numbers of roots along with their lengths were measured, as well as fresh weights of shoot and root, and then plants were kept in oven for 7 days at $70{ }^{\circ} \mathrm{C}$ and again dry weights were measured. Hydrogen peroxide in the leaf samples was determined at an absorbance of $390 \mathrm{~nm}$ using a UV-Vis spectrophotometer (IRMECO U-2020) according to the method described by Velikova et al. [25]. MDA was measured at two wavelengths of $532 \mathrm{~nm}$ and 600 $\mathrm{nm}$ [26]. Total soluble proteins were estimated using the dye-binding method described by Bradford [27].

\subsection{Antioxidant Enzymes Activities}

Fresh leaves $(0.25 \mathrm{~g})$ were finely ground under chilled conditions in $5 \mathrm{~mL}$ of phosphate buffer (50 $\mathrm{mM}$ with $\mathrm{pH}$ 7.8) for the extraction of antioxidant enzymes. Centrifugation of the mixture was performed at $12,000 \times \mathrm{g}$ for $20 \mathrm{~min}$ at $4{ }^{\circ} \mathrm{C}$. The supernatant was re-centrifuged at $15,000 \times \mathrm{g}$ for $10 \mathrm{~min}$ and then the resultant extract stored at $-20{ }^{\circ} \mathrm{C}$ for determining the activity of antioxidant enzymes. The activity of SOD was determined following the method 
described by Giannopolitis and Ries [28] by determining the enzyme inhibition of photochemical reduction of nitroblue tetrazolium (NBT). Then absorbance was read at $560 \mathrm{~nm}$ with a UV-visible spectrophotometer (Hitachi U-2100, Tokyo, Japan). Both CAT and POD were assayed according to the procedure described by Chance and Maehly [29]. The activity of all antioxidant enzymes was determined on protein basis.

\subsection{Statistical Analysis}

The design of the experiment was completely randomized factorial and each treatment was replicated thrice. The data were subjected to statistical analysis using COSTAT computer software.

\section{Results}

\subsection{Growth Characteristics}

\subsubsection{Shoot Length}

Maize hybrids treated with thiourea and salt resulted in non-significant differences $(P>0.05)$ in both seasons. Hybrids (H), salt (S) and thiourea (TU) independently as the main factors significantly $(P<0.01)$ affected shoot length in both seasons (Fig. 1a).

In autumn, hybrid 33M15 performed relatively better than DK6789 (2\%) when treated with $400 \mu \mathrm{M}$ thiourea under salt stress. Thiourea increased the shoot length of $33 \mathrm{M} 15$ and DK6789 up to $27 \%$ and 53\%, respectively, as compared to their controls. In spring season, salt stress reduced the shoot length in both hybrids (24\% reduction in 33M15 and 12\% reduction in DK6789). However, in spring season the application of $400 \mu \mathrm{M}$ thiourea was markedly effective in improving the shoot length in both the hybrids under salt stress (44\% and 56\% in 33M15 and DK6789, respectively). The differences in the shoot length of two maize hybrids were $6 \%$ and $2 \%$ under control or salt stress conditions (33M15 > DK6789) with the application of thiourea.

\subsubsection{Root Length}

Maize hybrids in both seasons when treated with thiourea and salt stress resulted in non-significant differences $(P>0.05)$. While $\mathrm{TU} \times \mathrm{H}$ treatments as the main factors significantly $(P<0.01)$ affected the root length of both maize hybrids (Fig. 1b).

In autumn season, hybrid 33M15 performed better than DK6789 (2.2\%) when treated with $400 \mu \mathrm{M}$ thiourea under $120 \mathrm{mM} \mathrm{NaCl}$. Salt stress reduced the root length of 33M15 (4\%) and increased the root length of DK6789 (5\%). Under non-saline condition, the root length of 33M15 increased by $<1.27 \%$ with the medium supplementation of thiourea as compared to sensitive hybrid. Thiourea increased the root length of $33 \mathrm{M} 15$ and DK6789 up to $28 \%$ and $44 \%$, respectively, as compared to their respective controls under salt stress. In spring, salt stress increased root length of 33M15 (1\%) and reduced the root length of DK6789 (1\%). On the other hand, the application of $400 \mu \mathrm{M}$ thiourea significantly enhanced the root length in both the hybrids under salt stress $(43 \%$ and $22 \%$ in 33M15 and DK6789, respectively). A comparison of hybrids data showed that both hybrids behaved differently. The differences in the root length of two maize hybrids were $9 \%$ and $21 \%$ under control and salt stress (33M15 > DK6789) with the application of thiourea.

\subsubsection{Number of Roots per Plant}

Maize hybrids in both seasons when treated with thiourea and salt stress resulted in non-significant differences $(P>0.05) . \mathrm{H} \times \mathrm{TU}$ significantly $(P<0.01)$ affected root number in autumn, while all three factors as main factors independently significantly $(P<0.01)$ affected root number in spring (Fig. 1c).

In autumn, hybrid 33M15 performed better $(20 \%)$ than DK6789 for number of roots when treated with $400 \mu \mathrm{M}$ thiourea under non-stressed condition, and $33 \mathrm{M} 15$ performed (12\%) better than DK6789 with thiourea application under salt stress condition. Salt stress affected the root number (increase 4\% of $33 \mathrm{M} 15$ and decrease $3 \%$ of DK6789). Thiourea increased the number of roots of 33M15 and DK6789 up to $18 \%$ and $23 \%$, respectively, under salt stress condition. For spring season, comparing hybrids data 


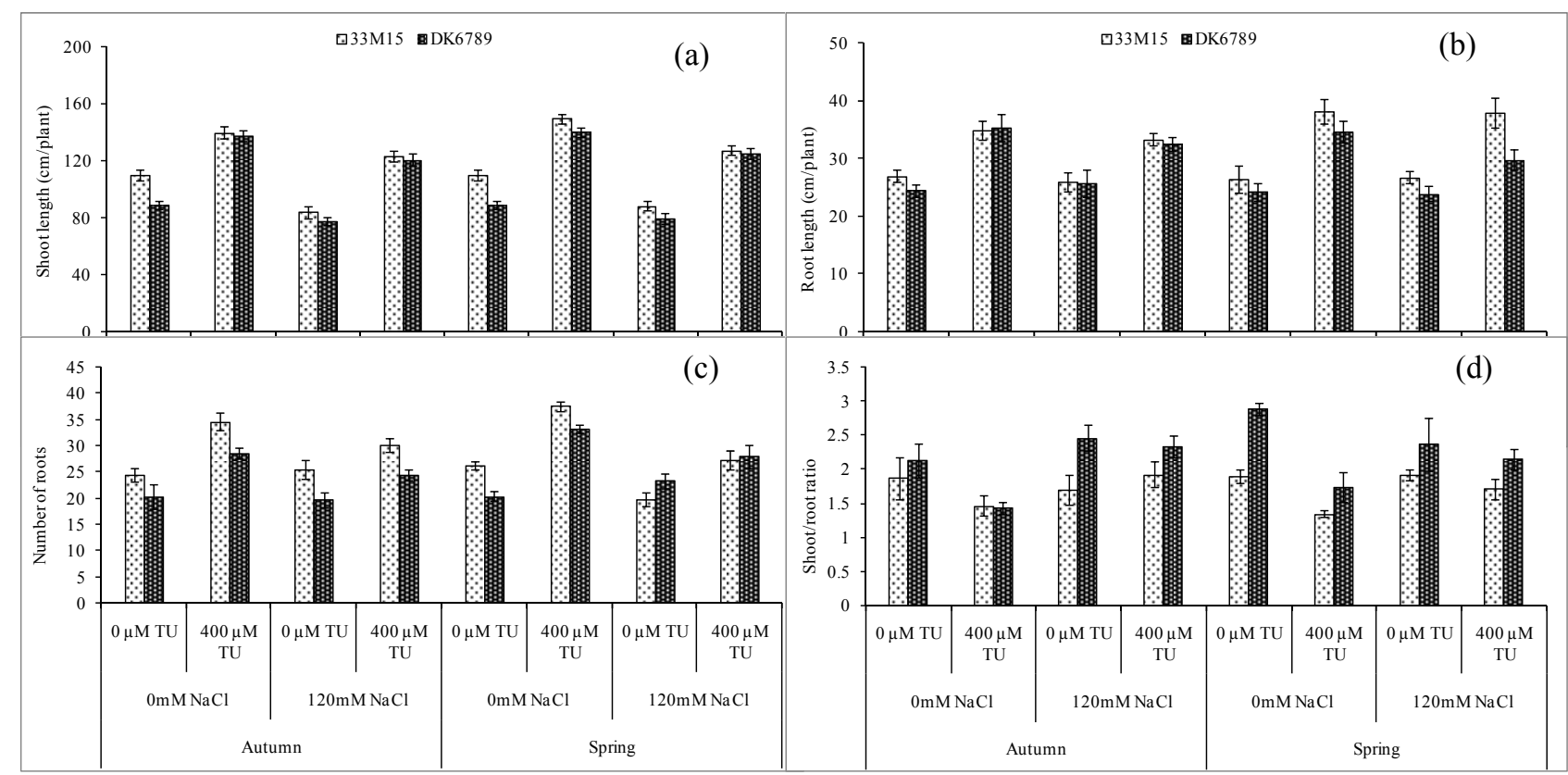

Fig. 1 Changes in shoot and root length, number of root, shoot/root ratio of maize hybrids under salt stress and thiourea (TU) application in alleviating salt stress toxicity in autumn and spring seasons.

showed that both hybrids performed differently. Salt stress decreased the root number of 33M15 (31\%) and increased the root number of DK6789 (12\%).

Thiourea $(400 \mu \mathrm{M})$ was proved to be effective in improving number of roots in both hybrids under stressed condition (19\% and 37\%, respectively). The differences in root number of two maize hybrids were $11 \%$ and $2.4 \%$ under control and salt stress (33M15 > DK6789) with the application of thiourea.

\subsubsection{Shoot/Root Ratio}

Maize hybrids in both seasons when treated with thiourea and salt stress resulted in non-significant differences $(P>0.05)$. While these three factors (independently) significantly affected the shoot/root ratio significantly $(P<0.01)$ in spring (Fig. 1d).

Salt stress made $9 \%$ decrease and $13 \%$ increase in shoot/root ratio of 33M15 and DK6789, respectively. In autumn, hybrid 33M15 performed better (1.7\% and $21 \%$, respectively) than DK6789 in autumn for the shoot/root ratio when treated with $400 \mu \mathrm{M}$ thiourea under stress and non-stressed condition. Thiourea increased the shoot/root ratio of 33M15 and DK6789 up to $13 \%$ and $4 \%$, respectively, under salt stress condition. In spring season, it was shown that both hybrids behaved differently in spring to the applied treatments. The differences in shoot/root ratio of two maize hybrids were $29 \%$ and $25 \%$ under control and salt stress (33M15 > DK6789) with the application of thiourea. The shoot/root ratio increased $1 \%$ in $33 \mathrm{M} 15$ and decreased $22 \%$ in DK6789 by the effect of salt stress.

\subsubsection{Shoot Fresh Weight}

In both seasons, maize hybrids treated with thiourea and salt stress resulted in non-significant $(P>0.05)$ differences, except $\mathrm{S}$ and TU (independently) as main factors affected significantly $(P<0.01)$ shoot fresh weight of both hybrids (Fig. 2a).

Salt stress reduced shoot fresh weight $(66 \%$ of $33 \mathrm{M} 15$ and $34 \%$ for DK6789). In autumn season, better results were found for 33M15 (9\%) than DK6789 (6\%) when treated with $400 \mu \mathrm{M}$ thiourea under stressed condition. Thiourea application significantly improved fresh weight of both hybrids under stress (4\% and 14\%, respectively). But application of thiourea increased the shoot fresh weight of 33M15 and DK6789 up to $102 \%$ and $66 \%$, respectively, under non-stressed conditions as compared to their controls. In spring season, the shoot 
fresh weight of the both hybrids was enhanced by 400 $\mu \mathrm{M}$ thiourea under salt stress $(120 \mathrm{mM} \mathrm{NaCl})$. Salt stress alone reduced the shoot fresh weight in both the hybrids (44\% reduction in 33M15 and $8 \%$ reduction in DK6789). However, the application of $400 \mu \mathrm{M}$ thiourea improved this attribute in both hybrids under control condition (113\% and $218 \%$, respectively). The differences in the shoot fresh weight of two maize hybrids were $32 \%$ and $30 \%$ under control and salt stress (33M15 > DK6789) with the application of thiourea.

\subsubsection{Root Fresh Weight}

Maize hybrids in both seasons treated with thiourea and salt stress resulted in non-significant differences $(P>0.05)$, although $\mathrm{H}, \mathrm{S}$ and $\mathrm{TU}$ (independently) exerted significant effects $(P<0.01)$ on root fresh weight of both maize hybrids (Fig. 2b).

Salt stressed reduced root fresh weight of $67 \%$ and $60 \%$ in both hybrids, respectively. In autumn season, hybrid 33M15 performed better $(5 \%$ and $28 \%$, respectively) than DK6789 for root fresh weight attribute when treated with $400 \mu \mathrm{M}$ thiourea under stress and non-stressed condition in autumn season. Thiourea increased the root fresh weight of 33M15 and DK6789 up to $100 \%$ and $45 \%$, respectively, as compared to their controls under salt stress condition. In spring season, salt stress caused substantial reduction in root fresh weight of both hybrids $(63 \%$ reduction in 33M15 and 66\% reduction in DK6789). Application of $400 \mu \mathrm{M}$ thiourea level significantly enhanced the root fresh weight in both hybrids under salt stress (33\% and 93\% in 33M15 and DK6789, respectively). Data showed that both the hybrids performed differently. The differences in root fresh weight of two maize hybrids were $9 \%$ and $14 \%$ under control and salt stress with the application of thiourea.

\subsubsection{Shoot Dry Weight}

In both seasons, the maize hybrids treated with thiourea and salt stress resulted in non-significant $(P>$ 0.05 ) differences on shoot dry weight. $\mathrm{H}, \mathrm{S}$ and $\mathrm{TU}$ treatments (independently) significantly $(P<0.01)$ affected root fresh weight of both hybrids (Fig. 2c).

In autumn, hybrid 33M15 performed better than DK6789 and showed more shoot dry weight of 11\% under stress, and DK6789 performed 16\% better than $33 \mathrm{M} 15$ under non-stressed condition with the medium supplementation of thiourea. Salt stress significantly reduced shoot dry weight of both hybrids $(29 \%$ and $33 \%$, respectively). But application of thiourea increased the shoot dry weight of 33M15 and DK6789 up to $75 \%$ and $57 \%$, respectively. In spring season, on the contrary, salt stress substantially reduced the shoot dry weight, but $400 \mu \mathrm{M}$ thiourea markedly enhanced shoot dry weight of both hybrids. Reduction in this attribute under salt stress was $28 \%$ in $33 \mathrm{M} 15$ and $42 \%$ reduction in DK6789. However, application of 400 $\mu \mathrm{M}$ thiourea improved shoot dry weight in both the hybrids under control condition (49\% in both hybrids). The differences in the shoot dry weight of two maize hybrids were $20 \%$ and $22 \%$ under control and salt stress $(33$ M15 > DK6789) with the application of thiourea.

\subsubsection{Root Dry Weight}

There were non-significant differences $(P>0.05)$ for all interactions, except $\mathrm{TU} \times \mathrm{S}$ interaction resulted in significant $(P<0.01)$ results on root dry weight. While $\mathrm{H}, \mathrm{S}$ and $\mathrm{TU}$ treatments (independently) affected significantly $(P<0.01)$ the root dry weight of both maize hybrids in both seasons (Fig. 2d).

In autumn, salt stress significantly reduced root dry weight $20 \%$ and $53 \%$ in 33M15 and DK6789, respectively. On the other hand, thiourea significantly improved this attribute $54 \%$ and $64 \%$ in $33 \mathrm{M} 15$ and DK6789, respectively. Hybrid 33M15 performed better than DK6789 when treated with $(400 \mu \mathrm{M})$ thiourea, $27 \%$ better under stressed condition and $18 \%$ better than control condition. In spring season, on the other hand, salt stress reduced root dry weight of both hybrids $(30 \%$ reduction in $33 \mathrm{M} 15$ and $13 \%$ reduction in DK6789). However, root dry weight of both hybrids 


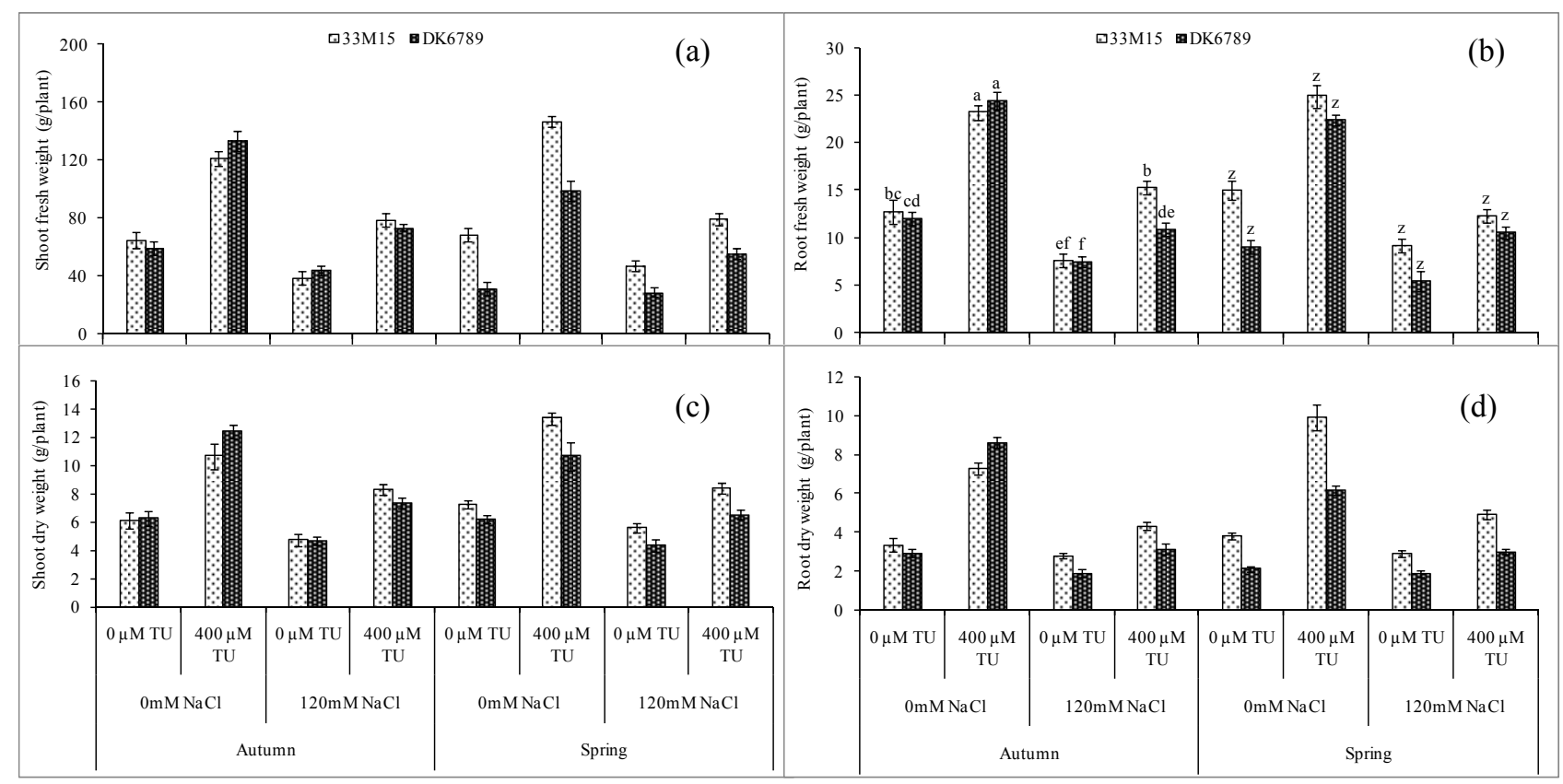

Fig. 2 Changes in shoot and root fresh weight, and shoot and root dry weight of maize hybrids (Zea mays L.) under salt stress and thiourea (TU) application in alleviating salt stress toxicity in autumn and spring seasons.

The bars labeled with alphabets show significant difference $(P<0.01)$.

was increased with $400 \mu \mathrm{M}$ thiourea medium supplementation under salt stress $(120 \mathrm{mM} \mathrm{NaCl})(68 \%$ and $60 \%$ in $33 \mathrm{M} 15$ and DK6789, respectively). The differences in root dry weight of two maize hybrids were $38 \%$ and $38.2 \%$ under salt stress $(33 \mathrm{M} 15>$ DK6789) without thiourea medium supplemented.

\subsection{Biochemical Attributes}

\subsubsection{Shoot $\mathrm{H}_{2} \mathrm{O}_{2}$}

Maize hybrids treated with thiourea under salt stress resulted in non-significant differences $(P>0.05)$ for all interactions in both seasons, except $\mathrm{TU} \times \mathrm{S}$. Individually, $\mathrm{H}, \mathrm{S}$ and $\mathrm{TU}$ exerted highly significant effects $(P<0.01)$ on shoot $\mathrm{H}_{2} \mathrm{O}_{2}$ (Fig. 3a).

In autumn, salt stress significantly enhanced accumulation of shoot $\mathrm{H}_{2} \mathrm{O}_{2}$ in both hybrids ( $81 \%$ and $84 \%$ in $33 \mathrm{M} 15$ and DK6789, respectively). In autumn, hybrid 33M15 performed relatively better than DK6789 with (31\%) less accumulation of $\mathrm{H}_{2} \mathrm{O}_{2}$ when treated with thiourea and salt. Thiourea decreased $\mathrm{H}_{2} \mathrm{O}_{2}$ of $33 \mathrm{M} 15$ and DK6789 up to $28 \%$ and $19 \%$, respectively, as compared to their controls under stressed condition. Salt stress enhanced the shoot $\mathrm{H}_{2} \mathrm{O}_{2}$ in both hybrids ( $82 \%$ in $33 \mathrm{M} 15$ and $83 \%$ in DK6789) in spring. However, the application of $400 \mu \mathrm{M}$ thiourea was markedly effective in improving the shoot $\mathrm{H}_{2} \mathrm{O}_{2}$ in both the hybrids under salt stress $(20 \%$ and $19 \%$ in $33 \mathrm{M} 15$ and DK6789, respectively). The differences in the shoot $\mathrm{H}_{2} \mathrm{O}_{2}$ of two maize hybrids were $2 \%$ and $13 \%$ under control and salt stress with the application of thiourea.

\subsubsection{Root $\mathrm{H}_{2} \mathrm{O}_{2}$}

Maize hybrids in both seasons when treated with thiourea under salt stress resulted in non-significant differences $(P>0.05) . \mathrm{H}, \mathrm{S}$ and TU (individually) exerted highly significant effects $(P<0.01)$ on shoot $\mathrm{H}_{2} \mathrm{O}_{2}$ (Fig. 3b).

Salt stress significantly enhanced accumulation of root $\mathrm{H}_{2} \mathrm{O}_{2}(81 \%$ and $81 \%$ in $33 \mathrm{M} 15$ and DK6789, respectively). Hybrid 33M15 performed better than DK6789 in autumn, 3\% when treated with thiourea under stress and $10 \%$ when treated with $400 \mu \mathrm{M}$ thiourea under $0 \mathrm{mM} \mathrm{NaCl}$. Thiourea caused decline in the root $\mathrm{H}_{2} \mathrm{O}_{2}$ of $33 \mathrm{M} 15$ and DK6789 up to $12 \%$ and $5 \%$, respectively, as compared to their controls under salt stress condition with thiourea application. 
In spring, salt stress increased $\mathrm{H}_{2} \mathrm{O}_{2}$ in both hybrids (81\% in 33M15 and $80 \%$ in DK6789). On the other hand, thiourea significantly reduced the $\mathrm{H}_{2} \mathrm{O}_{2}$ in both the hybrids under salt stress $(12 \%$ and $4 \%$ in $33 \mathrm{M} 15$ and DK6789, respectively). While comparing hybrids, data showed that both hybrids performed differently. The differences in the root $\mathrm{H}_{2} \mathrm{O}_{2}$ of two maize hybrids were $3 \%$ and $10 \%$ in control condition and under salt stress with the application of thiourea.

\subsubsection{Shoot MDA}

When maize hybrids treated with thiourea under salt stress, there were significant $(P<0.05)$ differences in autumn and non-significant $(P>0.05)$ in spring. All factors (individually) affected significantly $(P<0.01)$ shoot MDA of both hybrids in both seasons (Fig. 3c).

Salt stress significantly enhanced accumulation of MDA $(33 \%$ and $43 \%$ in 33M15 and DK6789, respectively). In autumn season, better results were found for 33M15 than DK6789 (17\% and 5\%) when treated with $400 \mu \mathrm{M}$ thiourea under stressed and non-stressed condition in autumn season. Salt stress significantly enhanced MDA, but thiourea improved shoot MDA of 33M15 and DK6789 (5\% and 23\%, respectively) as compared to their controls. In spring season, data showed that shoot MDA of both the hybrids was controlled by thiourea under salt stress $(120 \mathrm{mM} \mathrm{NaCl})$. Salt stress without thiourea enhanced the shoot MDA in both the hybrids (58\% and $39 \%$ in $33 \mathrm{M} 15$ and DK6789, respectively), thiourea reduced it by $33 \%$ in $33 \mathrm{M} 15$ and $18 \%$ in DK6789. The differences in the shoot MDA of two maize hybrids were $92 \%$ and $52 \%$ under control condition and salt stress with the application of thiourea.

\subsubsection{Root MDA}

Maize hybrids in both seasons when treated with thiourea under salt stress resulted in non-significant differences $(P>0.05)$, except $\mathrm{S} \times \mathrm{TU}$ interaction significantly affected $(P<0.01)$ root MDA. While $\mathrm{H}$, $\mathrm{S}$ and TU treatments independently exerted significant effects $(P<0.01)$ on the root MDA (Fig. 3d).

Salt stress caused huge accumulation of root MDA (53\% and $65 \%$ in 33M15 and DK6789, respectively). Hybrid 33M15 performed better (11\%) than DK6789

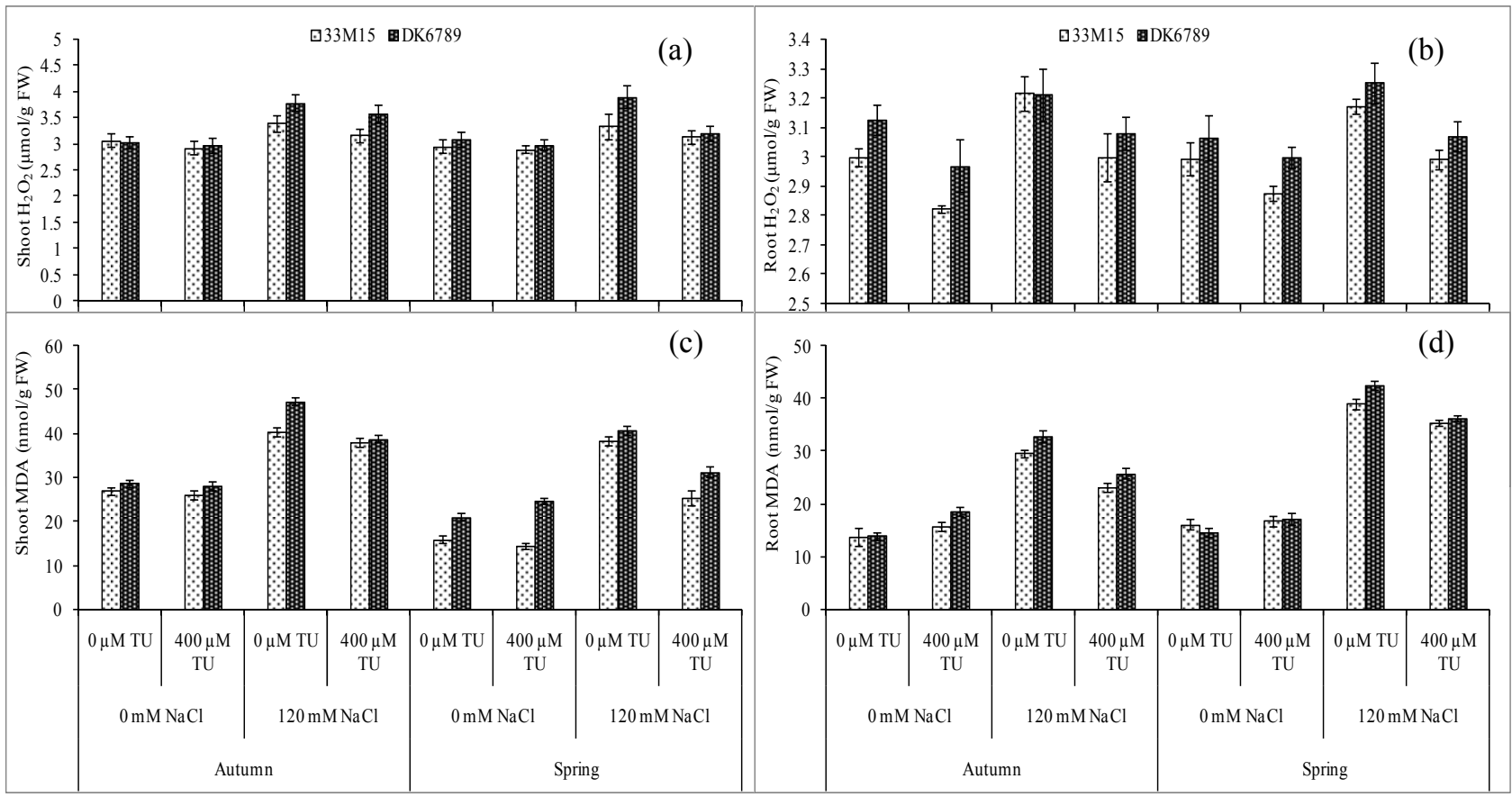

Fig. 3 Changes in $\mathrm{H}_{2} \mathrm{O}_{2}$ and MDA in root and shoot of maize hybrids (Zea mays L.) under salt stress and thiourea (TU) application in alleviating salt stress toxicity in autumn and spring seasons.

The bars labeled with alphabets show significant difference $(P<0.01)$. 
under stress, and DK6789 performed better (26\%) than 33M15 under non-stressed condition when treated with $400 \mu \mathrm{M}$ thiourea in autumn season. Thiourea decreased the root MDA of $33 \mathrm{M} 15$ and DK6789 up to $21 \%$ and $13 \%$, respectively, as compared to their controls under salt stress condition. Salt stress enhanced root MDA (58\% and $57 \%$ in 33M15 and DK6789, respectively), on the other hand, thiourea caused huge reduction in root MDA of both hybrids (9\% in 33M15 and 21\% in DK6789) in spring. Comparing hybrids data showed differences in root MDA of two maize hybrids $11 \%$ under control and $26 \%$ under salt stress with the application of thiourea.

\subsubsection{Shoot SOD}

The response of maize hybrids when treated with thiourea and salt stress resulted in non-significant effects $(P>0.05)$ in autumn season and significant effects $(P<0.01)$ in spring season, and the main factors TU, $\mathrm{S}$ and $\mathrm{H}$ (individually) significantly $(P<$ 0.01) affected SOD in both seasons (Fig. 4a).

In autumn, salt stress markedly enhanced SOD activity in both hybrids (23\% in 33M15 and $18 \%$ in DK6789). Hybrid 33M15 performed better than DK6789 when treated with thiourea under non-stressed (7\%) and stressed (29\%) condition, respectively. Salt stress significantly enhanced SOD of both hybrids, and thiourea further enhanced SOD activity (55\% and $7 \%$, respectively). In spring season, salt stress enhanced SOD in both hybrids (19\%), and thiourea application further increased SOD activity (48\% upgraded in 33M15 and 27\% in DK6789). The difference in the SOD of two maize hybrids was 34\% and $23 \%$ with and without salt stress with the application of thiourea.

\subsubsection{Shoot POD}

Maize hybrids in both seasons when treated with thiourea and salt stress resulted in non-significant differences $(P>0.05)$, while main factors TU, $\mathrm{S}$ and $\mathrm{H}$ (individually) significantly $(P<0.01)$ affected SOD in both seasons (Fig. 4b).

In autumn season, hybrids accumulated huge POD under salt stress (13\% in 33M15 and 12\% in DK6789). In comparison, hybrids 33M15 performed better (4.1\%) than DK6789 for POD when treated with 400 $\mu \mathrm{M}$ thiourea under non-stressed condition, and 33M15 performed better (11.5\%) than DK6789 with thiourea application under stressed condition. Thiourea increased the POD of 33M15 and DK6789 up to 35\% and $28 \%$, respectively, under salt stress condition. Salt stress caused huge increase in POD activity (23\% in $33 \mathrm{M} 15$ and 35\% in DK6789). Comparing hybrids data showed that both hybrids performed differently. The difference in POD of two maize hybrids was $27 \%$ and $17 \%$ under control and salt stress with the application of thiourea in spring season. In spring, thiourea increased the POD of 33M15 and DK6789 up to $17 \%$ and $23 \%$, respectively, under salt stress condition. Thiourea $(400 \mu \mathrm{M})$ was proved to be effective in improving POD in both hybrids in both seasons. Hybrid 33M15 responded better in spring season.

\subsubsection{Shoot CAT}

Maize hybrids in both seasons when treated with thiourea and salt stress resulted in non-significant differences $(P>0.05)$, but $\mathrm{H} \times \mathrm{S}$ and $\mathrm{S} \times \mathrm{TU}$ showed significant $(P<0.01)$ results. While main factors TU, $\mathrm{S}$ and $\mathrm{H}$ (individually) significantly $(P<0.01)$ affected CAT in both seasons (Fig. 4c).

Salt stress significantly enhanced activity of CAT (29\% in 33M15 and 3.6\% in DK6789). In autumn, hybrids performed differently (33M15 > DK6789), and hybrid $33 \mathrm{M} 15$ performed better $(21 \%$ and $6 \%$, respectively) than DK6789 for shoot CAT when treated with $400 \mu \mathrm{M}$ thiourea under stress and non-stressed condition. Thiourea increased the shoot CAT of 33M15 and DK6789 up to $36 \%$ and $65 \%$, respectively, under salt stress condition. In spring, salt stress increased accumulation of CAT (24\% in 33M15 and $14 \%$ in DK6789). Comparing hybrids, data showed that both hybrids performed differently in spring. The differences in shoot CAT of two maize hybrids were $17 \%$ and $39 \%$ under control and salt stress with the application of thiourea. 


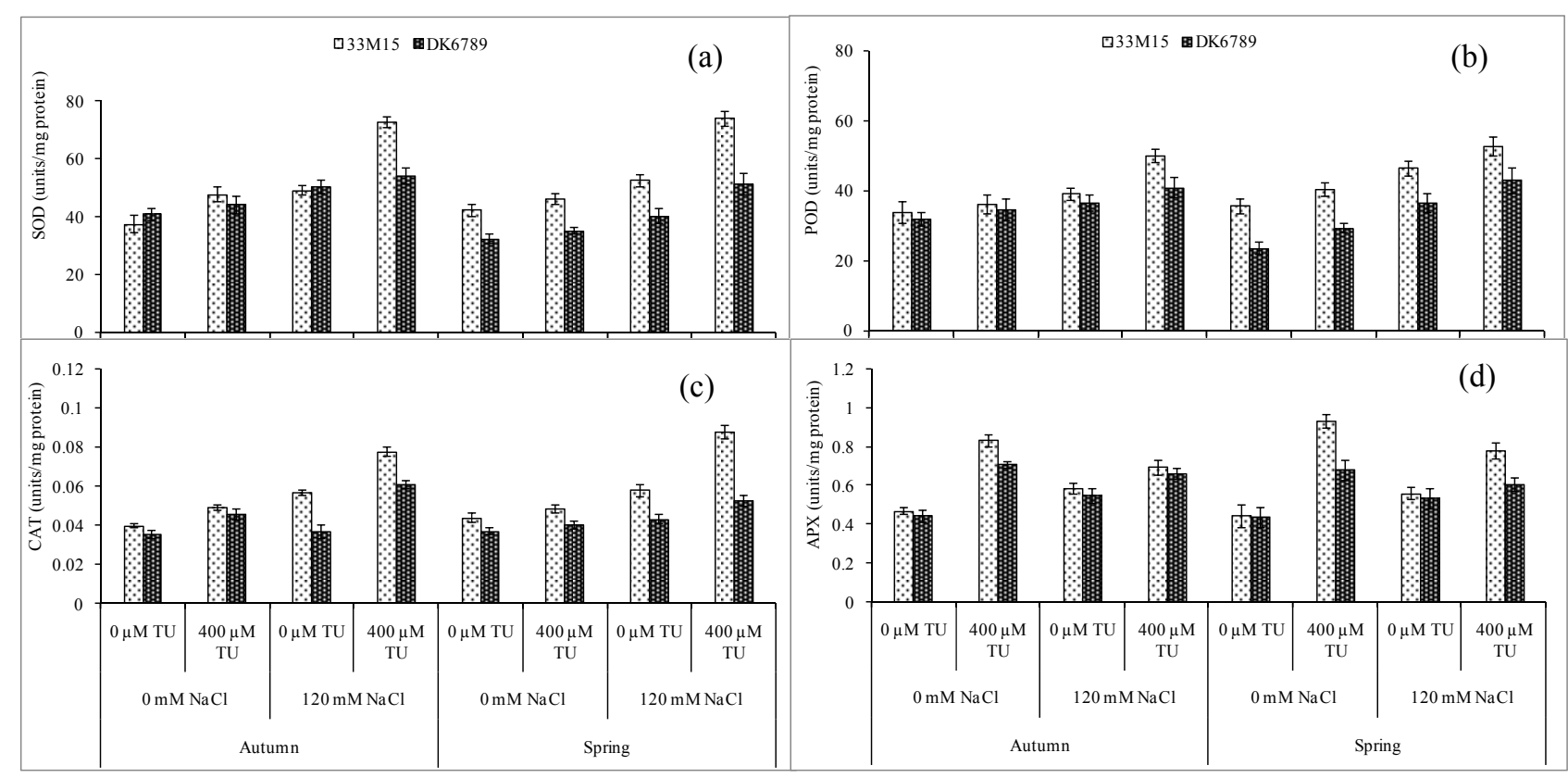

Fig. 4 Changes in SOD, POD, CAT and APX of maize hybrids (Zea mays L.) under salt stress and role of thiourea (TU) in alleviating salt stress toxicity in autumn and spring seasons.

The bars labeled with alphabets show significant difference $(P<0.01)$.

\subsubsection{Shoot Ascorbate Peroxidase (APX)}

When maize hybrids treated with thiourea and salt stress resulted in non-significant differences $(P>$ 0.05), except $\mathrm{H} \times \mathrm{S}$ and $\mathrm{S} \times \mathrm{TU}$, but $\mathrm{H} \times \mathrm{TU}$ exerted significant $(P<0.01)$ effects as the main factors on shoot APX in both seasons (Fig. 4d).

Salt stress enhanced (19\% in 33M15 and $18 \%$ in DK6789) APX content. Comparing hybrids when treated with $400 \mu \mathrm{M}$ thiourea 33M15 performed relatively better than DK6789 under stress (4.8\%) and non-stressed $(15 \%)$ condition in autumn. Thiourea increased the APX of 33M15 and DK6789 up to 18\% and $20 \%$ under stress, respectively (Fig. 4a). Salt stress enhanced APX in both hybrids $(20 \%$ accumulation in 33M15 and 18\% increase in DK6789) in spring. Though, medium supplementation thiourea markedly enhanced APX in both hybrids under salt stress $(39 \%$ and $13 \%$ in 33M15 and DK6789, respectively). Differences in shoot APX of two maize hybrids were $27 \%$ and $22 \%$ under normal and stressed condition with thiourea application. Spring season was better with higher antioxidants activities in both hybrids.
Under all treatments and both seasons, the response of 33M15 was markedly better than DK-6789.

\section{Discussion}

Exogenously applied growth regulators induce cell divisions, and ultimately increase shoot and root length [30]. In this study, the selected level of thiourea $(400 \mu \mathrm{M})$ was used for managing salinity toxicity of maize hybrids [31]. Salt stress reduced shoot and root length, number of roots, shoot/root ratio, fresh and dry weight of shoot and root (Figs. 1 and 2), while medium complimented thiourea proved to be very effective in partially nullifying the damaging effect of salinity on both hybrids. As a strategy of alleviating the adverse effects of salinity, the exogenous supply of growth bio-regulators, such as glycinebetaine, proline and trehalose has been in vogue for quite some time with great success $[14,32]$. There are different facets of salinity damage on plant cells and tissues, but the oxidative damage has been given due consideration [14], while thiourea has been reported to reduce the salinity damage [17]. 
The salinity-induced disturbances taking place in leaf tissues are due to over-accumulation of some toxic ions, particularly $\mathrm{Na}^{+}$and $\mathrm{Cl}^{-}$are most abundant, which result in generation of ROS, like $\mathrm{H}_{2} \mathrm{O}_{2}[33,34]$. In this study, salinity stress caused the enhanced production of $\mathrm{H}_{2} \mathrm{O}_{2}$ and MDA in the roots and leaves of both hybrids in both seasons, although substantial differences were noticed under salinity stress (Figs. 1a and $1 b$ ). Increased amount of $\mathrm{H}_{2} \mathrm{O}_{2}$ and MDA under salinity stress is the result of excessive concentration of toxic ions in tissues, which alter the biochemical mechanisms in a way that the membranes of the cellular compartments where the ROS metabolism takes place are specifically damaged [35]. In tobacco, salt stress induced higher ROS production as well as increased membrane lipid peroxidation [36]. In this study, although there was enhanced production of $\mathrm{H}_{2} \mathrm{O}_{2}$ and MDA, the medium supplementation of thiourea markedly reduced their tissue concentration.

To cope with the enhanced production of ROS, the plants have natural tendency of induction of enzymatic antioxidants, which successfully douse the ROS and partially rescue the plants from adversaries of salinity [14]. It is not clear thus far that whether thiourea has the tendency to stimulate the induction of antioxidants. The presence of thiol functional groups in the thiourea molecules may allow such an action of thiourea. The results of the present study revealed that medium supplementation of thiourea substantially reduced the production of ROS with a greatest enhancement in the activity of SOD, followed by CAT and POD. The dismutation of superoxide radicals is an important step in the reduction of oxidative damage to the cells [37]. Thus, it can be concluded that the medium supplementation of thiourea can partially alleviate the salinity-induced production of ROS in maize, although the response of hybrids was markedly different.

Prevailing seasonal conditions have great influence on the plant performance, which is the result of the operation of internal mechanisms [38]. This study revealed that spring season was more profound than autumn season for maize growth irrespective of the treatments applied. During spring season, the temperature is within the moderate range, which favors plant growth. The tissue accumulation of toxic ions is relatively lesser than that observed in the autumn season. The use of thiourea was even more effective in the spring than the autumn season. Thus, it can be asserted that the use of thiourea for the improvement of salinity induced oxidative damage can be more effective in the spring season.

\section{Conclusions}

Salinity stress declined the growth attributes of both maize hybrids, although differentially. Enhanced production of $\mathrm{H}_{2} \mathrm{O}_{2}$ and MDA was the result of salinity-induced damage on the plant tissues (root and leaves). Medium supplementation of thiourea improved the growth of both maize hybrids by reducing the synthesis of ROS and MDA, indicating a possible repair mechanism operated by MDA. Seasonal conditions do influence the production of ROS and efficacy of thiourea application. For achieving better salinity tolerance, the supplementation of thiourea may be made in the spring season.

\section{Acknowledgments}

This study is a part of Ph.D. research work of Ph.D. scholar Miss Tayyaba Sanaullah PIN No. Batch-VIIBm7-063, which is funded by Higher Education Commission (HEC), Pakistan through indigenous Ph.D. scheme.

\section{References}

[1] Shahbaz, M., Ashraf, M., Al-Qurainy, F., and Harris, P. J. C. 2012. "Salt Tolerance in Selected Vegetable Crops." Crit. Rev. Plant Sci. 31 (4): 303-20.

[2] Flowers, T. J., Galal, H. K., and Bromham, L. 2010. "Evolution of Halophytes: Multiple Origins of Salt Tolerance in Land Plants." Funct. Plant Biol. 37: 604-12.

[3] Akram, N. A., and Ashraf, M. 2011. "Improvement in Growth, Chlorophyll Pigments and Photosynthetic 
Performance in Salt-Stressed Plants of Sunflower (Helianthus annuus L.) by Foliar Application of 5-Aminolevulinic Acid." Agrochimic 55: 94-104.

[4] Mittova, V., Guy, M., Tal, M., and Volokita, M. 2004. "Salinity Up-Regulates the Antioxidative System in Root Mitochondria and Peroxisomes of the Wild Salt Tolerant Tomato Species Lycopersicon pennellii.” J. Exp. Bot. 55 (399): 1105-13.

[5] Almeselmani, M., Deshmukh, P. S., Sairam, R. K., Kushwaha, S. R., and Singh, T. P. 2006. "Protective Role of Antioxidant Enzymes under High Temperature Stress." Plant Sci. 171 (3): 382-8.

[6] Noreen, S., Ashraf, M., Hussain, M., and Jamil, A. 2009. "Exogenous Application of Salicylic Acid Enhances Antioxidative Capacity in Salt Stressed Sunflower (Helianthus annuus L.) Plants.” Pak. J. Bot. 41: 473-9.

[7] Noreen, Z., Ashraf, M., and Akram, N. A. 2010. "Salt-Induced Regulation of Some Key Antioxidant Enzymes and Physio-Biochemical Phenomena in Five Diverse Cultivars of Turnip (Brassica rapa L.).” J. Agron. Crop Sci. 196 (4): 273-85.

[8] Raza, S. H., Ashraf, M., Athar, H. R., and Hameed, A. 2007. "Glycinebetaine-Induced Modulation of Antioxidant Enzyme Activities and Ion Accumulation in Two Wheat Cultivars Differing in Salt Tolerance." Environ. Exp. Bot. 60: 368-76.

[9] Noreen, Z., and Ashraf, M. 2009. "Assessment of Variation in Antioxidative Defense System in Salt Treated Pea (Pisum sativum L.) Cultivars and Its Putative Use as Salinity Tolerance Markers." J. Plant Physiol. 166 (16): 1764-74.

[10] Farooq, M., Basra, S. M. A., Wahid, A., and Rehman, H. 2009. "Exogenously Applied Nitric Oxide Enhances the Drought Tolerance in Fine Grain Aromatic Rice (Oryza sativa L.).” J. Agron. Crop Sci. 195 (4): 254-61.

[11] Srivastava, A. K., Ramaswamy, N. K., Mukhopadhyaya, R., Chiramal-Jincy, M. G., and D’Souza, S. F. 2009. "Thiourea Modulates the Expression and Activity Profile of mtATPase under Salinity Stress in Seeds of Brassica juncea." Ann. Bot. 103 (3): 403-10.

[12] Wahid, A., Parveen, M., Gelani, S., and Basra, S. M. A. 2007. "Pretreatment of Seeds with $\mathrm{H}_{2} \mathrm{O}_{2}$ Improves Salt Tolerance of Wheat Seedling by Alleviation of Oxidative Damage and Expression of Stress Proteins." J. Plant Physiol. 164 (3): 283-94.

[13] Wahid, A., Sehar, S., Perveen, M., Gelani, S., Basra, S. M. A., and Farooq, M. 2008. "Seed Pre-treatment with Hydrogen Peroxide Improves Heat Tolerance in Maize at Germination and Seedling Growth Stages." Seed Sci. Technol. 36 (3): 633-45.

[14] Perveen, A., Wahid, A., Mahmood, S., Hussain, I., and Rasheed, R. 2015. "Possible Mechanism of
Medium-Supplemented Thiourea in Improving Growth, Gas Exchange and Photosynthetic Pigments in Cadmium Stressed Maize (Zea mays).” Brazilian Journal of Botany 38 (1): 71-9.

[15] Desikan, R., Cheung, M. K., Clarke, A., Golding, S., Sagi, M., Fluhr, R., Rock, C., Hancock, J., and Neill, S. 2004. "Antioxidant Responses of Rice Seedlings to Salinity Stress." Plant Sci. 135: 1-9.

[16] Kumawat, P. D. 2004. "Response of Indian Mustard (Brassica juncea (L.) Czern. and Coss.) to Seed Treatment and Foliar Spray of Sulphuric Acid under Varying Levels of Irrigation." Ph.D. thesis, Rajasthan Agricultural University, Bikaner.

[17] Anjum, F., Wahid, A., Farooq, M., and Javed, F. 2011. "Potential of Foliar Applied Thiourea in Improving Salt and High Temperature Tolerance of Bread Wheat (Triticum aestivum)." Int. J. Agric. Biol. 13: 251-6.

[18] Zia, A., Guo, B., Ullah, I., Ahmad, R., Khan, M. A., Abbasi, B. H., and Wei, Y. 2011. "Salinity Tolerance and Site of $\mathrm{K}^{+}$Accumulation in Four Maize Varieties Grown in Khyber Pakhtoonkhwa Region of Pakistan." J. Med. Plants Res. 5 (25): 6040-7.

[19] Aghaleh, M., Niknam, V., and Ebrahimzadeh, H. 2009. "Salt Stress Effect on Growth, Pigments, Proteins and Lipid Peroxidation in Salicornia persica and S. europaea." Biol. Plant. 53 (2): 243-8.

[20] Jaleel, C. A., Riadh, K., Gopi, R., Manivannan, P., Inès, J., AI-Juburi, H. J., Zhao, C. X., Shao, H. B., and Rajaram, P. 2009. “Antioxidant Defense Responses: Physiological Plasticity in Higher Plants under Abiotic Constraints." Acta Physiol. Plant. 31 (3): 427-36.

[21] Joseph, B., and Jini, D. 2011. "Development of Salt Stress-Tolerant Plants by Gene Manipulation of Antioxidant Enzymes.” Asian J. Agric. Res. 5 (1): 17-27.

[22] Perveen, A., Wahid, A., Hussain, I., Rasheed, R., and Mahmood, S. 2013. "Growth Bio-regulatory Role of Root-Applied Thiourea: Changes in Growth, Toxicity Symptoms and Photosynthetic Pigments of Maize." Pak. J. Agric. Sci. 50: 455-62.

[23] Noctor, G., Mhamdi, A., and Foyer, C. H. 2014. "The Roles of Reactive Oxygen Metabolism in Drought: Not So Cut and Dried." Plant Physiol. 164 (4): 1636-48.

[24] Hoagland, D. R., and Arnon, D. I. 1950. The Water-Culture Method for Growing Plants without Soil. Berkeley, Calif: College of Agriculture, University of California.

[25] Velikova, V., Yordanov, I., and Edreva, A. 2000. "Oxidative Stress and Some Antioxidant Systems in Acid Rain-Treated Bean Plants: Protective Role of Exogenous Polyamines." Plant Sci. 151: 59-66.

[26] Carmak, I., and Horst, J. H. 1991. "Effects of Aluminum on Lipid Peroxidation, Superoxide Dismutase, Catalase 
and Peroxidase Activities in Root Tips of Soybean (Glycine max)." Physiol. Plant. 83 (3): 463-8.

[27] Bradford, M. M. 1976. "A Rapid and Sensitive Method for the Quantitation of Microgram Quantities of Protein Utilizing the Principle of Protein-Dye Binding." Ann. Biochem. 72: 248-54.

[28] Giannopolitis, C. N., and Ries, S. K. 1977. "Superoxide Dismutase: Part I, Occurrence in Higher Plants." Plant Physiol. 59 (2): 309-14.

[29] Chance, B., and Maehly, A. C. 1955. "The Assay of Catalase and Peroxidase." Methods in Enzymol. 2: 764-817.

[30] Silveira, V., Santa-Catarina, C., Tun, N. N., Scherer, G. F. E., Handro, W., Guerra, M. P., and Floh, E. I. S. 2006. "Polyamine Effects on the Endogenous Polyamine Contents, Nitric Oxide Release, Growth and Differentiation of Embryogenic Suspension Cultures of Araucaria angustifolia (Bert.) O. Ktze..” Plant Sci. 171: 91-8.

[31] Sanaullah, T., Wahid, A., Javed, F., and Sadia, B. 2016. "Optimization of Thiourea Level at Cellular and Whole Plant Level for Maize Hybrids (Zea mays L.)." Applied Ecology Environ. Research 14 (5): 1-18.

[32] Francisco, A. A., Tavares, A. R., Kanashiro, S., Ramos, P. R. R., and Lima, G. P. P. 2008. "Plant Growth Regulators in Polyamines Endogenous Levels during the Development of Taro Cultivated in Vitro." Ciencia Rural 38 (5): 1251-7.

[33] Mittler, R., Vanderauwera, S., Gollery, M., and Breusegem, F. V. 2004. "Reactive Oxygen Gene Network of Plants." Trends Plant Sci. 9 (10): 490-8.

[34] Sanaullah, T., Wahid, A., Javed, F., and Sadia, B. 2016. "Enhanced Osmolyte Accumulation Counteracts Salt Stress by Controlled ROS: Evidence from Biochemical Studies.” Inter. J. Develop. Research 6 (7): 8667-75.

[35] Apel, K., and Hirt, H. 2004. "Reactive Oxygen Species: Metabolism, Oxidative Stress and Signal Transduction." Annu. Rev. Plant Biol. 55: 373-99.

[36] Katsuhara, M., Otsuka, T., and Ezaki, B. 2005. "Salt Stress-Induced Lipid Peroxidation Is Reduced by Glutathione S-Transferase, But This Reduction of Lipid Peroxides Is Not Enough for a Recovery of Root Growth in Arabidopsis.” Plant Sci. 169 (2): 369-73.

[37] Rahnama, H., and Ebrahimzadeh, H. 2005. "The Effect of $\mathrm{NaCl}$ on Antioxidant Enzyme Activities in Potato Seedlings." Biol. Plant. 49 (1): 93-7.

[38] Taiz, L., Zeiger, E., Moller, I. M., and Murphy, A. 2015. Plant Physiology and Development, 6th ed.. Sunderland, MA: Sinauer Associates, Inc.. 